\title{
As dificuldades vivenciadas pelos profissionais de enfermagem no atendimento pré-hospitalar
}

\section{The difficulties experienced by nursing professionals in prehospital care}

\author{
Diogo Rodrigues Canesin' •Vinicius de Lima Lovadini • Sabrina Ramires Sakamoto ${ }^{3}$
}

\begin{abstract}
RESUMO
Introdução: O Serviço de Atendimento Móvel de Urgência (SAMU) é responsável por oferecer um atendimento precoce a vítima após ter ocorrido algum agravo a sua saúde, proporcionando um atendimento adequado e de qualidade no local em que o usuário se encontra e o transporte precoce ao local mais adequado à resolução do problema de saúde até os serviços de saúde fixos, hierarquizado e integrado ao Sistema Único de Saúde (SUS). Existem inúmeras situação que podem atrapalhar o atendimento, como locais imprevisíveis, materiais e recursos humanos limitados e apropriados para garantir a assistência de saúde a vítima, podendo afetar diretamente a qualidade do atendimento prestado. Objetivo: Compreender as dificuldades vivenciadas pela enfermagem no atendimento pré-hospitalar. Métodos: Estudo qualitativo, não experimental, exploratório descritivo transversal realizado através entrevista semiestruturada gravada com os profissionais de enfermagem do SAMU de Araçatuba-SP. Resultados: Participaram do estudo 16 profissionais de enfermagem do SAMU, a maioria do sexo feminino, com idade média de 38 anos, metade dos participantes eram enfermeiros e outra metade técnicos de enfermagem do serviço. Os discursos foram submetidos à Análise de Conteúdo de Bardin, emergindo nas categorias: Desconhecimento populacional da função do SAMU; Dificuldades com a Central de Regulação; Estratégias de melhorias para o serviço. Conclusão $O$ estudo permitiu a reflexão dos profissionais acerca da atuação no serviço, instigando as reflexões apresentando possíveis estratégias para melhoria da realidade.
\end{abstract}

Palavras-chave: Assistência Pré-hospitalar; Urgência. Enfermagem em Emergência; Serviços Médicos de Emergência.

\begin{abstract}
Introduction:The Mobile Emergency Care Service (SAMU) is responsible for providing early care to the victima after an injury to their health, providing adequate and quality care where the useris and early transportation to the victim. Most appropriate place to solve the health problem to the fixed health services, hierarchized and integrated in to the Unified Health System (SUS). There are numerous situations that may disrupt care, such as unpredict table locations, limited and appropriate human resources and resources to ensure healthcare for the victim, and may directly affect the quality of care provided. Objective:To understand the difficulties experienced by nursing in prehospital care. Methods: Qualitative, non -experimental, descriptive cross-sectional exploratory study conducted through through semi-structured interview recorded with the nursing professionals of SAMU of Araçatuba-SP. Results: The study included 16 SAMU nursing professionals, mostly female, with an average age of 38 years, half of the participants were nurses and the other half nursing technicians of the service.The speeches were submitted to Bardin's ContentAnalysis, emerging in the categories: Population ignorance of SAMU function; Difficulties with the Regulatory Center; Improvement strategies for the service. Conclusion:The study allowed the reflection of the professionals about the performance in the service, instigating the reflections presenting possible strategies to improve the reality.
\end{abstract}

Keywords: Prehospital Care; Urgency; Nursing in Emergency; Emergency Medical Services.

2 Enfermeiro, Mestre em Ciência Animal pela Universidade Estadual Paulista (UNESP), Araçatuba Especialização em andamento em Saúde Pública com ênfase em Saúde da Família pela FMU, Doutorando em Enfermagem pela Escola de Enfermagem de Ribeirão Preto da Universidade de São Paulo-(EERP-USP). ORCID ID https://orcid.org/0000-000I-9066-2160

3 Enfermeira, Mestra em Enfermagem pela Universidade Estadual Paulista (UNESP), Botucatu, Especialista em Urgência e Emergência, Saúde Pública, Docência e Enfermagem do Trabalho. Doutoranda em Enfermagem pela Universidade Estadual Paulista (UNESP), Botucatu. ORCID ID https://orcid.org/0000-0003-0I89-7043 


\section{INTRODUÇÃO}

Situações de emergências em saúde são aquelas nas quais o atendimento deve ser imediato, enquanto situações classificadas como urgências são situações em que podem ser prestadas em tempo não superior a duas horas. As condições não urgentes são definidas como aquelas que podem ser encaminhadas a um pronto atendimento ambulatorial, ou para $\circ$ atendimento ambulatorial convencional (1).

O serviço de Atendimento Pré-Hospitalar (APH) é definido como uma assistência realizada às pessoas em situações de agravos urgentes nas próprias cenas em que os eventos ocorrem, contribuindo para um atendimento precoce ${ }^{(2)}$. $O$ atendimento pré-hospitalar vem cada vez mais se aprimorando, de maneira indispensável, caracterizado como um diferencial, contribuindo para salvar inúmeras vítimas e melhor prognóstico dos sobreviventes atendidos (3).

O Serviço de Atendimento Móvel de Urgência (SAMU), foi idealizado na França, em I 986 como Service d'AideMédicale d'Urgence. No Brasil o Governo Federal instituiu a Portaria GM N ${ }^{\circ}$ I.864, de 29 de setembro de 2003, referente a Política Nacional de Atenção às Urgências, a ser implantada em todas as unidades federadas ${ }^{(4)}$. Essa portaria determinou o financiamento para investimento e custeio do componente pré-hospitalar móvel, em municípios e regiões de todo o território brasileiro, caracterizado como um serviço gratuito, criado para atendimento pré-hospitalar ${ }^{(5)}$.

O objetivo do SAMU é oferecer um atendimento precoce a vítima após ter ocorrido algum agravo a sua saúde, este agravo pode ser de natureza clínica, cirúrgica, traumática, obstétrica, pediátrica, psiquiátrica entre outras, proporcionando um atendimento adequado e de qualidade no local em que o usuário se encontra e o transporte precoce ao local mais adequado à resolução do problema de saúde, se necessário, de forma segura até os serviços de saúde fixos, hierarquizado e integrado ao Sistema Único de Saúde (SUS), que possam melhor atende-lo, respondendo de forma resolutiva às necessidades e garantindo a continuidade do cuidado ${ }^{(4-6-7-8)}$. A criação do SAMU auxilia consideravelmente na redução do número de óbitos, complicações e/ou lesões permanentes decorrentes da falta de socorro precoce, diminuindo assim o tempo de internação nos hospitais ${ }^{(9)}$.

Tendo como estrutura de funcionamento uma rede de Atenção às urgências o SAMU é constituído por diversos profissionais como: médicos, enfermeiros, técnicos de enfermagem, condutores, telefonistas auxiliares de regulação médica e rádio operadores. Para que esta rede de atenção tenha uma eficácia no seu atendimento é necessária uma base descentralizada pautada em uma infraestrutura que garanta tempo resposta de qualidade, regional ou sediado em municípios de grande extensão territorial e/ou baixa densidade demográfica conforme definido no plano regional com a configuração mínima necessária para abrigo, alimentação, conforto das equipes e estacionamentos das ambulâncias ${ }^{(10)}$.

Após o acolhimento e identificação dos chamados, acionado por meio de telefonia de discagem rápida, número 192, as solicitações são analisadas pelo médico regulador onde classifica o nível de urgência de cada solicitação, definindo qual será o recurso necessário para o atendimento, que pode envolver desde uma simples orientação médica até o envio de uma unidade ao local (4).

O SAMU atua 24 horas por dia, está estruturado com duas modalidades de unidades móveis: Suporte Avançado à Vida (SAV) e unidades móveis de Suporte Básico de Vida (SBV). O SAV é unidade destinado ao atendimento e transporte de pacientes de alto risco em emergências, tem como características manobras invasivas, de maior complexidade, é conduzida por três profissionais, sendo um motorista, um enfermeiro e um médico.A ambulância de SBV é um veículo destinado a preservação da vida, para transporte de pacientes, não classificado com potencial de necessidade de intervenção médica, é conduzida por dois profissionais, sendo um o motorista e um técnico ou auxiliar de enfermagem ${ }^{(8-11)}$.

O enfermeiro desempenha um papel fundamental durante $o$ atendimento pré-hospitalar, regulado pelo Conselho Federal de Enfermagem (COFEN), por meio da Resolução $n^{\circ} 375 / 2011$, que dispõem sobre a presença do enfermeiro no APH e Inter Hospitalar, em situações de risco conhecido ou desconhecido ${ }^{(12)}$. Atua através da assistência direta ao paciente tanto no suporte de vida como nas ações gerenciais que contribuem para que o atendimento seja prestado com segurança e qualidade. Participa e coordena procedimentos que visam à estabilização do quadro clínico do paciente, realizando seu transporte, para que receba um tratamento adequado. Devido ao grau de complexidade das ações, existe a necessidade de que o profissional enfermeiro adquira conhecimentos técnicos e científicos, aperfeiçoando sua os procedimentos executados ${ }^{(13)}$.

Para que isso ocorra de forma rápida e satisfatória, surge a necessidade de veículos móveis adaptados com instrumentos e aparelhos que proporcionem condições para a equipe do SAMU realizarem um atendimento eficaz, seguro e tranquilo e, caso necessário, o deslocamento para o centro de atendimento hospitalar de referência ${ }^{(9)}$.

Cada atendimento realizado pela equipe do SAMU é único, apresentando variáveis distintas, destacando a atenção da equipe de forma ágil e eficiente. Existem inúmeras situação que podem atrapalhar o $\mathrm{APH}$, demandando da 
equipe conhecimento, habilidades e atitudes inesperadas, como $o$ atendimento em locais imprevisíveis, materiais e recursos humanos limitados e apropriados para garantir a assistência de saúde a vítima, podendo afetar diretamente a qualidade do atendimento prestado ${ }^{(14)}$.

O SAMU é considerado um serviço complexo e de grande importância para sociedade, possuindo como objetivo maior o atendimento ágil de vítimas no local da ocorrência, buscando reduzir a mortalidade, tempo de internação e as complicações em consequência a falta de atendimento imediato ${ }^{(9)}$.

A APH deve ser realizada por profissionais capacitados, treinados, com competência técnica, capazes de oferecer ao paciente procedimentos específicos de acordo com sua gravidade, justifica-se pesquisar os desafios vivenciados pelos enfermeiros para assistirem o paciente em risco de morte ${ }^{(13)}$.

Diante dessas considerações, a relevância do estudo é reconhecida buscando compreender as dificuldades vivenciadas pela enfermagem no atendimento pré-hospitalar, instigando a reflexão sobre a assistência do cuidado na APH, assim considerando possíveis estratégias para melhoria da realidade investigada. $O$ objetivo desse estudo foi compreender as dificuldades vivenciadas pela enfermagem no atendimento pré-hospitalar.

O estudo teve como o objetivo evidenciar quais as dificuldades encontradas pelos profissionais de enfermagem do SAMU no atendimento pré-hospitalar.

\section{MATERIAL E MÉTODOS}

Tratou-se de uma pesquisa de campo, de caráter exploratório, com delineamento transversal e prospectivo com análise qualitativa descritiva dos dados.

Foram entrevistados dezesseis colaboradores do Samu sendo nove mulheres e sete homens.

Foram adotados como critérios de inclusão, os profissionais presentes no local nas datas de coleta. Foram excluídos da pesquisa: os profissionais em férias ou afastados por motivos de saúde.

A coleta de dados ocorreu após aprovação do Comitê de Ética e Pesquisa, parecer $n^{\circ}$ 3.425.622, e aprovação pelo sujeito da pesquisa por meio da anuência no Termo de Consentimento Livre Esclarecido, mantendo sigilo sobre as informações obtidas dos sujeitos, conforme Resolução $N^{\circ} 466 / 2012$, da Comissão Nacional de ética e Pesquisa (CONEP) vinculada ao Ministério da Saúde.

Foi utilizado para a coleta de dados, entrevista semiestruturada e gravada, na qual foi realizado análise segundo (13), no qual só foram aplicados nos sujeitos da pesquisa mediante apresentação explícita da pesquisa bem como dos propósitos de estudo. A realização da entrevista ocorreu de forma individual, com resposta espontânea para análise das informações investigadas, dentre elas so- cioeconômicas, tais como: idade, grau de escolaridade, estado civil, além de questões relacionadas, especificamente. Manterá o anonimato dos entrevistados, sendo eles identificados pela letra "E" para enfermeiro e "TE" para técnico de enfermagem, seguidos do algarismo numérico correspondente à ordem da entrevista $(E I, E 2$, TEI,TE2...).

A análise dos dados foi realizada por meio do programa Microsoft Excel 2007 e as variáveis do estudo foram apresentadas por meio da estatística descritiva evidenciando a distribuição relativa (\%) e absoluta (n) dos dados e posteriormente apresentada na forma de tabelas.

\section{RESULTADOS}

Participaram do estudo 16 profissionais de enfermagem atuantes no SAMU de Araçatuba, a maioria do sexo feminino (56\%), com idade média de 38 anos (33-52 anos), metade dos participantes são católico, a maioria casados (69\%), com ensino superior completo (69\%) e metade dos participantes são enfermeiros e técnicos de enfermagem do serviço. Estes dados estão descritos na tabela I.

As entrevistas, com duração média de 3 minutos, foram realizadas na base do SAMU de Araçatuba em local reservado, proporcionando um ambiente organizativo e calmo, favorecendo a concentração para resposta. As falas das participantes, foram trabalhadas de acordo com ${ }^{(14)}$, dando origem a três categorias que contemplam as dificuldades vivenciadas pela enfermagem no atendimento pré-hospitalar.

Posteriormente a gravação de cada uma das entrevistas, foi realizado a transcrição na íntegra. $O$ primeiro contato com as entrevistas referiu de uma leitura superficial, com vistas a aproximação com o conteúdo de cada fala das participantes, seguida de leituras exaustivas, com o intuito de identificação dos núcleos de sentido e elaboração das seguintes categorias: Desconhecimento populacional da função do SAMU; Dificuldades com a Central de Regulação; Estratégias de melhorias para o serviço. Descritos abaixo com as entrevistas dos profissionais de enfermagem.

\section{(I) Desconhecimento populacional da função do SAMU}

Os sujeitos do estudo referiram em suas falas que a população na maioria das vezes desconhece a real função do SAMU, dessa forma acionando o serviço sem legitima necessidade, justificando que muitas das ocorrências recebidas diariamente não correspondem ao atendimento de urgência e emergência. Os profissionais surpreendem-se muitas vezes ao chegar ao local de destino, pois se deparam com casos clínicos dos mais variados, como pode ser visto nas falas seguintes: 
TABELA 1 - Distribuição dos participantes da pesquisa, segundo características sociodemográficas no município de Araçatuba-SP, Brasil, 2019.

\begin{tabular}{|c|c|c|c|}
\hline Variável & Categoria & $\mathbf{N}$ & $\%$ \\
\hline \multirow{2}{*}{ Sexo } & Masculino & 7 & 44 \\
\hline & Feminino & 9 & 56 \\
\hline \multirow{3}{*}{ Idade } & 30-35 anos & 5 & 31 \\
\hline & $35-40$ anos & 7 & 44 \\
\hline & $<40$ anos & 4 & 25 \\
\hline \multirow{3}{*}{ Religião } & Católico & 8 & 50 \\
\hline & Evangélico & 4 & 25 \\
\hline & Outra & 4 & 25 \\
\hline \multirow{3}{*}{ Estado Civil } & Casado & 11 & 69 \\
\hline & Solteiro & 3 & 19 \\
\hline & Divorciado & 2 & 12 \\
\hline \multirow{2}{*}{ Escolaridade } & Ensino Superior & 11 & 69 \\
\hline & Ensino Médio & 5 & 31 \\
\hline \multirow{2}{*}{ Categoria Profissional } & Enfermeiro & 8 & 50 \\
\hline & Técnico de enfermagem & 8 & 50 \\
\hline
\end{tabular}

Fonte: Dados da Pesquisa.

"A população desconhece quando tem que chamar o SAMU [...]." (TE3)

"[...] a família apresenta estrutura e condições de locomover o mesmo, pois o paciente está orientado, deambulando, não apresenta alterações em sinais vitais e a familia tem automóvel na garagem [...]." (TEI)

"[...] chama por coisas desnecessárias e acabam influenciando o atendimento de outras pessoas que podem realmente estar precisando do serviço." (E4)

"[...]não sabe como funciona, a gente chega no local fica bravo porque o médico começa a perguntar um monte de coisas, onde se viu o médico perguntar um monte de coisa e não mandar logo a ambulância, começa aí a falta de informação que falta para população, nem é culpa deles, é falta de informação mesmo." (E5)

"[...] falta de cultura e conhecimento da população, de quando haver o acionamento da equipe, em que situações, qual a real função do SAMU, porque a gente acaba tendo uma equipe empenhada para atividades que não são da $A P H$, as vezes realizando apenas o transporte de pacientes que poderia ser destinada a outra instituição [...]. ”(E6)

"[...] respeito da população, respeitar a equipe em si e os procedimentos que são feitos, hoje todo mundo tem celular, tem informação e as vezes acaba até intimidando a equipe durante $o$ atendimento. $A$ falta de conhecimento, de conscientização do que é o SAMU e como é feito o nosso trabalho, a gente faz toda uma parte de verificação de sinais vitais de regulação via fone, então as vezes a população não sabe disso acaba querendo interferir, acha que a gente está demorando e até não tem credibilidade até com o crescimento da equipe, não somos profissionais, nós estudamos para isso." (TE5)
"Essas dificuldades maiores são a respeito à população não saber ligara para o SAMU ou para o Bombeiro, saber a diferente entre um e outro. [...] Muitas das vezes agente depara com as duas viaturas na ocorrência que não teria necessidade. [...]. É um pouco cultural e um pouco falta de conhecimento dos serviços prestados à população. "(TE6)

Os relatos apresentam as dificuldades que os profissionais identificam como questão cultural ou desconhecida da população em acionar esse serviço, ocasionando inúmeras vezes em deslocamento desnecessário da equipe, citando o prejuízo ao usuário em situação real de emergência, podendo refletir na qualidade do sucesso dos atendimentos.

Outros profissionais apresentaram os curiosos e familiares no momento da ocorrência que interferem, tentando auxiliar o profissional, porém muitas vezes atrapalham o atendimento que está sendo realizado, essas situações estão descritas nos seguintes relatos:

"Durante o atendimento domiciliar também, as vezes estamos fazendo o atendimento domiciliar e os vizinhos pedem também que a gente verifique pressão, questionam muitas das vezes porque daquilo, atrapalhando nossa linha de raciocínio, de trabalho. Eles acabam querendo ajudar ou interferem querendo saber o que aconteceu e interferem, a gente acaba tendo uma limitação de espaço [..]. "(E2)

"[...] curiosos e familiares, quando a gente chega na residência, eles querem dizer o que o próprio profissional $A P H$ faz, porém isso acaba atrapalhando o nosso serviço no atendimento pré-hospitalar." (E3)

"[...] durante $o$ atendimento a gente não consegue se comunicar com o paciente e os familiares, os acompanhantes aí, 
mas não uma questão de não conseguir se comunicar por falta de falar, mas é porque as conversas não se encaixam mesmo, os profissionais não conseguem atingir o objetivo." (E4)

A comunicação entre o profissional, acompanhante/ familiar e paciente no momento da ocorrência foi descrito como uma limitação de entrosamento, dificultando o atendimento. Muitas vezes os presentes no momento da ocorrência querem auxiliar de maneira direta a equipe do SAMU, contudo neste momento a equipe necessita de espaço e colaboração de todos os envolvidos.

\section{(2) Dificuldades com a Central de Regulação}

Os indivíduos participantes do estudo expõem-se sobre o trabalho da Central de Regulação do serviço, citando alguns fatores que dificultar o trabalho rotineiro do SAMU, conforme os relatos abaixo.

"[...] acho que não investiga de forma aprofundada a ocorrência [...]. O que é passado pela regulação, muitas vezes a população desconhece a natureza da ocorrência e a regulação manda uma ambulância que não tem o suporte completo." (TE3)

"Na priorização dos atendimentos, você tem um deslocamento não adequado, não condizente com o tipo de recursos que você vai. De repente você tem uma ambulância com poucos recursos e pega um caso extremamente grave, uma ambulância de intermediária pega um caso leve e a avançada só sai para atendimentos, quando o paciente está entubado, já assistido para transferência, ou em outros casos só chega para constata óbito. " (EI)

"[...] desde a informação que o paciente passou para o médico, até o que o médico conseguiu absorver daquela informação e o que é passado para agente, muitas vezes a gente chega e não é nada o que foi passado para agente, a maioria das vezes acontece isso. "(E5)

"[...] falta de orientação adequada da equipe de regulação médica com relação ao deslocamento por meios próprios do paciente. "(TEI)

"Hoje a maior dificuldade, é relacionado a regulação médica, o tipo de atendimento, tipo de ambulância, qual suporte que será dado. "(TE5)

Nota-se claramente, através das falas mencionadas pelos profissionais de enfermagem da amostra, uma insatisfação com a Central de Regulação, referente as informações coletadas dos usuários durante a regulação e as viaturas estabelecidas para ocorrência.

\section{(I) Estratégias de melhorias para o serviço}

Nos relatos dos profissionais entrevistados, foi possível identificar sugestões de estratégias que possam meIhorar a qualidade e eficiência do serviço. Houve relatos referente a necessidade de educação continuada a população, através de palestras, esclarecimentos pelas mídias, como uma possível estratégia mobilizadora da comuni- dade, por meio da informação disseminada pode auxiliar na compreensão da população sobre função do SAMU e seu acionamento. A seguir descrito nos relatos:

"Eu acho que teria que ter alguém para dar orientação as pessoas da residência [...]. " (TE 2)

"A conscientização em relação $a$ isso e também a conscientização de um outro problema que a gente enfrenta que é quando acionar o SAMU, quando acionar o Corpo de Bombeiros, seria de grande utilidade, tanto para a população quanto principalmente para nos profissionais, que essa educação continuada a população fosse feita com publicidade, palestras, enfim, para que a população entenda o nosso trabalho, as nossas dificuldades e assim eles possam colaborar com a agente também, evitando trotes, evitando a curiosidade dentro do atendimento, evitando também os chamados desnecessários, que não são atendimentos de urgência e emergência, facilitando para a gente.” (E2)

"[...] principalmente aqui na nossa região que a população não sabe qual é o trabalho da parte do SAMU em si, que entra da parte de urgência e emergência, só seria triado paciente de urgência e emergência, e nem sempre é o foco do nosso trabalho aqui." (E3)

"O SAMU como educação continuada externa também deveria ter como palestras, orientando a população como é o serviço [...]. " (E5)

"[...] fazer um trabalho com mídia, internet, conscientizando isso, explicando para população o que é o SAMU, quando chamar o SAMU, o que é o bombeiro, quais são os profissionais que atuam, o que é competência deles, e o respeito a nos funcionários públicos. " (TE 5)

"Eu acho que um serviço de auditoria, a princípio e uma intervenção rápida de um órgão competente da secretaria de saúde [...] (TE8)

Em relação a equipe atuante no serviço, foi destacado por alguns profissionais participantes a necessidade de treinamentos e reuniões para melhorar o entrosamento da equipe e comunicação multiprofissional e com o usuário durante a regulação da ocorrência.

"[...] treinamento com a equipe para tentar entender esses aspectos, uma equipe melhor treinada com a situação, não que isso irá resolver de fato os problemas. "(E4)

"O protocolo protege ambos os lados, protege o paciente, a comunidade, a família e o profissional, então tem que gerar dados, o negócio é dados, o porquê, o quanto está saindo, o quanto está vindo, então tem que ser baseado por estatística, porque que determinado lugar demora para chegar, porque cada condutor segue um caminho diferente para ir, porque uns andam rápido, outros mais devagar, então tem um protocolo a seguir. [...].Tem que ter levantamentos de dados, depois fazer uma avaliação, avaliação mensal, trimestral, você vê algumas entidades, eles têm dados. [...].” (E7)

"[...] a regulação médica fazer questionamentos a família, se tem carro próprio, se tem meios de estar locomovendo 
a pessoa até o local e orientar com relação a necessidade da ambulância no local, que geralmente é para pacientes acamados, alterações visíveis de sinais vitais, que qualquer leigo percebe quando a pessoa está com uma dificuldade respiratória, ou uma fraqueza estrema que não consegue deambular." (TE I)

"[...] ter treinamentos médicos, como fazer uma boa regulação, que tipo de ambulância vai para cada caso o suporte que é dado. Retirar informações mais eficazes e não só ter o treinamento, mas a educação continuada, é um trabalho constante, um aperfeiçoamento que seja semestral, a cada mês, um trabalho, não isolado, algo contínuo. "(TE5)

\section{DISCUSSÃO}

De acordo com Decreto $n^{\circ} 7.508 / 2012$, o SAMU representa um importante porta de entrada do SUS, atendendo diversas demandas emergenciais de forma imediata a população realizando o atendimento inicial ao usuário. Caracterizado como um serviço de atendimento a vítimas em situações de urgência e emergência ${ }^{(16)}$.

Alguns dos relatos descritos nesse estudo foi demonstrado a dificuldade destacada pelos profissionais na assistência à população durante a ocorrência. Destacado o desconhecimento da população em relação a função do serviço, situação essa que pode levar ao deslocamento desnecessário da equipe, podendo prejudicar o atendimento a quem realmente encontra em alguma situação de emergência. $\mathrm{Na}$ literatura observa-se que outros estudos que realizam a análise da natureza das ocorrências atendidas pelo SAMU destacam a predominância de atendimento às ocorrências de natureza clínica (16-17-18-19).

Estudo concretizado em três diferentes municípios, dois do estado de São Paulo e um de Minas Gerais, com o objetivo de avaliar saberes de população acerca do SAMU apresentou o conhecimento da população razoável sobre o serviço, no entanto, existem problemas presentes no serviço relacionados principalmente à demanda inadequada, uma vez que a população é quem julga subjetivamente $o$ que considera urgente e aciona atendimento ${ }^{(17)}$.

Outro estudo realizado com a população em Porto Alegre apresentou as divergências entre a regulação e a percepção dos usuários sobre o funcionamento do serviço e significado de "risco de vida", esses fatores foram fortemente implicados na justificativa da demanda não pertinente presente no serviço local ${ }^{(18)}$.

O conhecimento da população sobre o SAMU é de extrema importância para que o usuário utilize de maneira consciente e eficaz o serviço, principalmente ao se deparar com uma situação de urgência e emergência. Alertando a população em relação a delonga ou mesmo o não acionamento desse serviço em casos emergenciais, sobrecarregando financeiramente o sis- tema e ocasionando sequelas ou diminuição da sobrevida das vítimas ${ }^{(18-19)}$.

$\mathrm{Na}$ segunda categoria foi possível observar as falas dos profissionais referentes aos chamados de socorros recebidos na Central de Regulação Médica do SAMU, relatado pelos participantes as divergências entre as regulações e as situações reais dos chamados. Estudo realizado no interior de São Paulo apresenta que a maioria dos pacientes atendidos estavam conscientes no momento da chegada da equipe do SAMU ao local da ocorrência, destacando que esse resultado pode evidenciar o fato de que muitos dos chamados de socorro recebidos na Central de Regulação Médica do SAMU podem não ser classificados como casos reais de urgência e emergência ${ }^{(19)}$.

A regulamentação dos serviços de APH no Brasil ocorre através da Portaria 2.048/GM, do Ministério da Saúde, assim denominando o APH móvel como SAMU. Este serviço deve ser entendido como uma atribuição da área de saúde, sendo vinculado a uma Central de Regulação, com equipe de profissionais, frotas de veículos compatíveis com as necessidades da população ${ }^{(8)}$.

Assim a Central de Regulação é responsável por prover um atendimento eficaz e adequado, compreende um processo de trabalho no qual realiza a escuta permanente pelo médico regulador, realizando o acolhimento, bem como o estabelecimento do ocorrido e uma estimativa inicial do grau de urgência de cada caso abordado, utilizando protocolos técnicos do serviço perante as respostas das ocorrências ${ }^{(19-20)}$.

A falta de compreensão da população reflete no processo de trabalho da regulação, intensificando a necessidade de compreensão desse processo, para agilizar o atendimento e atuação qualificada dos profissionais do SAMU.

$\mathrm{Na}$ terceira unidade de significado deste estudo foi apresentado as falas dos participantes referentes as sugestões para melhoria do serviço atual. Destaca-se principalmente as sugestões para orientação populacional, treinamento da equipe e comunicação multiprofissional.

Estudo apresenta destaque oferecer a comunidade em geral a educação em ralação a utilização adequada do SAMU, buscando proporcionar o reconhecimento das situações para acionar o serviço, também a capacidade de reconhecer rapidamente e corretamente os contextos que requerem $o$ atendimento do SAMU ${ }^{(5)}$.

Os profissionais de enfermagem têm papel de destaque no SAMU ao atuar, muitas vezes, em atividades que podem extrapolar a assistência aos usuários. Além disto, a importância da enfermagem neste processo está na sua competência e atuação em ações de educação a população e em primeiros socorros, participando de projetos Inter setoriais capazes de intervir na realidade atual ${ }^{(19)}$.

Estudo realizado com equipe do SAMU apresenta que 
a qualidade do atendimento está diretamente relacionada ao bom funcionamento da equipe, assim destaca-se a comunicação e integração durante o atendimento. As interversões prestadas pelo serviço configuram-se como um trabalho coletivo de cumplicidade e cooperação dos profissionais envolvidos ${ }^{(20)}$.

Durante esse processo de atendimento do SAMU é importante destacar a comunicação como um fator essencial para garantir o atendimento eficiente e eficaz, acontecendo constantemente, refletindo na motivação, cooperação e satisfação de toda equipe.

\section{CONCLUSÃO}

As dificuldades vivenciadas pela enfermagem no atendimento pré-hospitalar foi o desconhecimento populacional e as dificuldades com a Central de Regulação, também foi possível identificar sugestões de estratégias que possam melhorar a qualidade e eficiência do serviço como educação continuada a população, através de palestras, esclarecimentos pelas mídias, como uma possível estratégia mobilizadora da comunidade, por meio da informação disseminada pode auxiliar na compreensão da população sobre função do SAMU e seu acionamento. 


\section{REFERÊNCIAS BIBLIOGRÁFICAS}

1. Schiavon ICA.A triagem em serviço de emergência. [dissertação de mestrado]. Campinas (SP): 2006 Faculdade de Ciências Médicas da Universidade Estadual de Campinas.

2. Pereira WA da P, Lima MAD da S. O trabalho em equipe no atendimento pré-hospitalar à vítima de acidente de trânsito. Rev da Esc Enferm da USP. 2009;43(2):320-7.

3. Machado CV, Lima LD de, O'Dwyer G, Andrade CLT de, Baptista TW de F, Pitthan RGV, et al. Gestão do trabalho nas unidades de pronto atendimento: Estratégias governamentais e perfil dos profissionais de saúde. Cad Saude Publica. 2016;32(2):I-I4. Adão R de S, Santos MR dos. Atuação do enfermeiro no atendimento pré-hospitalar móvel TT - Desempeño del enfermero en la atención pre-hospitalaria móvil TT - Nurses performance in a mobile prehospital care. REME rev min enferm [Internet]. 20I2;16(4):60I-8. Available from: http://pesquisa.bvsalud.org/portal/resource/pt/lil-667724

4. Vieira CMS, Mussi FC. A implantação do projeto de atendimento Móvel de Urgência em Salvador/BA: panorama e desafios. Rev da Esc Enferm da USP. 2008;42(4):793-7.

5. Farmácia $C$ federal de. Resolução No586 De 29 De Agosto De 20I3.J Chem Inf Model. 20I3;20I3:I-I2.

6. Marques GQ, Da Silva Lima MAD, Ciconet RM. Agravos clínicos atendidos pelo serviço de atendimento móvel de urgência (SAMU) de Porto Alegre - RS. ACTA Paul Enferm. 201 I;24(2): I85-9|.

7. Brasil. Ministério da Saúde. Portaria n. 2048/GM, de 5 de novembro de 2002, dispõe sobre o regulamento técnico dos sistemas estaduais de urgência e emergência. 2002. Diário Oficial da República Federativa do Brasil. Brasília: Imprensa Oficial; novembro 2002.

8. Silva SF da, Lucio DBM, Ilha S, Diefenbach GD, Pereira JC. Difficulties Lived in an Urgency Mobile Service: the Nurse Team Perception. R Enfem Cent O Min. 2013;4(2): I I6I-72.

9. Malvestio MAA, Cardoso de Sousa RM. Suporte avançado à vida:Atendimento a vítimas de acidentes de trânsito. Rev Saude Publica. 2002;36(5):584-9.

10.CONSELHO FEDERAL DE ENFERMAGEM. Resolução 375 de 2011 de 22 de março de 201I. Dispõe sobre a presença do enfermeiro no atendimento pré-hospitalar e inter hospitalar, em situações de risco conhecido ou desconhecido. 201 I;2-3.
11. Ribeiro ÍAP, Da Mata KSS, Pereira KLA, Carvalho GCN, Macedo JB, Pereira PSL, et al. Entraves no atendimento pré-hospitalar do samu: percepção dos enfermeiros. Rev Enferm UFPE line. 20।8; I2(8):2। 37.

12.Santos FM. Resenha ANÁLISE DE CONTEÚDO:A VISÃO DE LAURENCE BARDIN. Rev Eletrônica Educ [Internet]. 2012;6(I):383-7. Available from: http://www.reveduc.ufscar.br/index.php/reveduc/article/viewFile/29//I56

13.Um EM, Atendimento SDE. Estressores E Coping Vivenciados Por Enfermeiros Stressors and Coping Experienced By Nurses in a Pre-Hospital Admission Care Delivery En Servicios De Atención Pre-Hospitalaria. Trauma. 2008; I3(I):33-43.

14. Machado CV, Salvador FGF, O’Dwyer G. Serviço de Atendimento Móvel de Urgência:Análise da política brasileira. Rev Saude Publica. 201 I;45(3):519-28.

15. Giaretta V, Ferronato M,Ascari T, Krauzer I. Profile of Events in a Mobile Emergency. Rev Baiana Enferm. 2012;26(2):478-87.

16. Garçon TAF, Aguiar LA, Nascimento ES do Voltarelli4A. Fatores desencadeantes de estresse do enfermeiro na unidade de urgência e emergência. Revista Enfermagem Atual [Internet]. I labr.20I9 [citado 2 I mar.2020];87(25). Available from: https://revistaenfermagematual.com.br/index.php/ revista/article/view/210

17. Cruz MC da, Camargo RP de, Ferreira AS, Borges MT, Cruz MCC da, Simonato LE. Conhecimentos sobre o serviço de atendimento móvel de urgência (SAMU) da população de três municípios com realidades distintas. Arch Heal Investig. 2017;6(6):269-74.

18. Samu S, I JDAP, Victor J, Costa G, I AAM. o Funcionamento do Serviço de Atendimento Móvel de Urgência ( Samu ) Knowlegdeof Medical Students about the. 20। 4;38(2):253-60.

19.BRASIL. Ministério da Saúde. Política Nacional de Atenção às Urgências [Internet]. Série E. Legislação de Saúde. 2006. I-I38 p. Available from: http://bvsms.saude.gov.br/bvs/publicacoes/politica_nacional_atencao_urgencias_3ed.pdf

20. Pereira WAP, Lima MADS. O Trabalho em equipe no atendimento pré-hospitalar à vítima de acidente de trânsito. RevEsc de Enferm USP; 2009; 42(2): 320-7.

Recebido: 2020-02-298

Aceito 2020-04-03 